\title{
LUCAT1 Epigenetically Downregulates the Tumor Suppressor Genes CXXC4 and SFRP2 in Gastric Cancer
}

\author{
Hyo Joo Byun ${ }^{1,2}$, Jung-Ho Yoon ${ }^{1}$, and Sang Kil Lee ${ }^{1,2}$ \\ ${ }^{1}$ Department of Internal Medicine, Institute of Gastroenterology, Yonsei University College of Medicine, Seoul; \\ ${ }^{2}$ Brain Korea 21 PLUS Project for Medical Science, Yonsei University, Seoul, Korea.
}

Purpose: The mechanisms of Wnt/ $\beta$-catenin pathway signaling and abnormal expression of tumor suppressor genes is not well known in gastric cancer (GC). Long non-coding RNA (lncRNA) has recently been identified as a possible link therein. In this study, we investigated the role of lung cancer associated transcript 1 (LUCAT1) in GC.

Materials and Methods: The expression of LUCAT1 in GC cell lines and 100 tissue samples was examined by qRT-PCR. Two different siRNAs were used for knockdown of LUCAT1 expression. Cell viability was assessed by MTT assay. To analyze metastasis, scratch wound-healing assay, a Matrigel invasion assay, and colony formation assay were performed. Apoptosis was analyzed by $\mathrm{PI}$ /Annexin-V staining. To check the methylation status in tumor suppressor genes, methylation-specific PCR was carried out. Western blot was performed to detect epithelial-mesenchymal transition and apoptosis markers upon silencing of LUCAT1 (siLUCAT1).

Results: LUCAT1 expression in GC cell lines and tissues was significantly elevated, compared to that in normal gastric cells and adjacent non-tumor tissues $(p<0.001)$. Two different siRNAs for LUCAT1 reduced cell proliferation, invasion, and migration, compared to siCT $(p<0.05)$, and these reductions were restored by pcDNA-LUCAT1 $(p<0.05)$. siLUCAT1 elicited upregulation of the expression of CXXC4 and SFRP2. The expression of H3K27me3 was reduced by siLUCAT1, and this reduction was correlated with methylation of CXXC4 and SFRP2. Inhibition of LUCAT1 up-regulated EZH2 expression and resulted in demethylation of CXXC4 and SFRP2 through the Wnt/ $\beta$-catenin signaling pathway.

Conclusion: We concluded that LUCAT1 induces methylation of $C X X C 4$ and SFRP2, thereby regulating Wnt/ $\beta$-catenin signaling in GC.

Key Words: Long non-coding RNA, LUCAT1, epigenetic modulation, gastric cancer

\section{INTRODUCTION}

Gastric cancer (GC) is the third leading cause of cancer-related

Received: August 25, 2020 Revised: September 2, 2020

Accepted: September 3, 2020

Corresponding author: Sang Kil Lee, MD, PhD, Division of Gastroenterology, Department of Internal Medicine, Institute of Gastroenterology, Yonsei University College of Medicine, 50-1 Yonsei-ro, Seodaemun-gu, Seoul 03722, Korea.

Tel: 82-2-2228-1996, Fax: 82-2-393-6884, E-mail: sklee@yuhs.ac

-The authors have no potential conflicts of interest to disclose.

(C) Copyright: Yonsei University College of Medicine 2020

This is an Open Access article distributed under the terms of the Creative Commons Attribution Non-Commercial License (https://creativecommons.org/licenses/ by-nc/4.0) which permits unrestricted non-commercial use, distribution, and reproduction in any medium, provided the original work is properly cited. deaths and is the most common gastrointestinal malignancy in East Asia, Eastern Europe, and parts of Central and South America. ${ }^{1,2}$ Epigenetic and genetic alterations account for a large proportion of carcinogenesis in various organs, including GC. ${ }^{3-5}$ Accumulation of multiple genetic alterations or epigenetic changes has been shown to be associated with dysregulation of oncogenes and tumor suppressor genes in GC. ${ }^{6,7}$

Alteration of the Wnt/ $\beta$-catenin signaling induces the accumulation and nuclear translocation of $\beta$-catenin, and is closely associated with the development and progression of GCs. ${ }^{8}$ The Wnt/ $\beta$-catenin signaling components, including the effectors and negative regulators, are often altered by epigenetic manners, including DNA methylation and histone modifications in various tumors. Meanwhile, research has shown that negative 
regulators of Wnt $/ \beta$-catenin signaling are important for tumor suppression: $C X X C 4$ (CXXC finger protein 4 ), a negative regulator of Wnt $/ \beta$-catenin signaling, is reduced by enhancer of zeste homolog 2 (EZH2) in GC ${ }^{8,9}$ EZH2 plays an important role in epigenetic regulation of the human genome and is known to be associated with the development of various cancers. ${ }^{10}$ Growing evidence has also indicated that Wnt/ $\beta$-catenin signaling components are regulated by not only long non-coding RNAs (lncRNAs) but also other noncoding classes. ${ }^{11-15}$

LncRNAs, non-coding RNA transcripts, contain more than 200 nucleotides that lack protein coding potential. ${ }^{16-18} \mathrm{LncRNAs}$ have been linked to a wide range of epigenetic alterations in various cancers, including chromatin-related changes, DNA methylation, and splicing control. ${ }^{17,19-24}$ Numerous IncRNAs have been found to contribute to cancer cell functions through silencing of tumor suppressors via interaction with EZH2. ${ }^{10} \mathrm{~A}$ significant number of lncRNAs have been reported to be involved in cancer development by inhibiting tumor suppressor genes through binding with EZH2. ${ }^{10,25,26}$ We recently reported that highly expressed IncRNA $^{12}$ in esophageal squamous cell carcinoma (HERES) regulate Wnt/ $\beta$-catenin signaling via interaction with EZH2. ${ }^{12}$

We previously reported that LUCAT1 promotes tumorigenesis by controlling the ubiquitination and stability of DNA methyltransferase 1 (DNMT1) in esophageal squamous cell carcinoma (ESCC). ${ }^{27}$ LUCAT1 activated DNMT1, a major DNA methylation protein, to repress the expression of tumor suppressor genes known to be involved in ESCC carcinogenesis. Since the publication of our report, LUCAT1 has been reported to be involved in the development of clear cell renal cell carcinoma, non-small lung cancer, glioma, osteosarcoma, and colorectal cancer. ${ }^{27-33}$

In this study, we investigated the functions and molecular mechanisms of LUCAT1 in the carcinogenesis of GC. The activation of EZH2 induced activation of the Wnt/ $\beta$-catenin pathway by repressing $C X X C 4$ expression in GC.$^{34}$ We also explored the interactions of LUCAT1 and EZH2, as well as regulatory networks of Wnt/ $\beta$-catenin signaling, ${ }^{8}$ with $C X X C 4$ and SFRP2 in GC.

\section{MATERIALS AND METHODS}

\section{Patients and tissue samples}

In total, $100 \mathrm{GC}$ tissue and paired adjacent gastric tissue samples were obtained from patients who underwent surgical resection for GC at Severance Hospital, Yonsei University College of Medicine. All samples were frozen in liquid nitrogen immediately after resection and stored at $-80^{\circ} \mathrm{C}$. All tissue samples were obtained after receiving written informed consent from patients according to the Declaration of Helsinki, and this study was approved by Institutional Review Board of the Yonsei University College of Medicine (\# 4-2011-0753).

\section{Cell lines and cell culture}

GC cell lines (AGS, MKN28, MKN45, MKN74, KATOIII) and the normal gastric cell line (GES1) were purchased from the Korean Cell Line Bank (KCLB, SNU, Seoul, Korea) and the American Type Culture Collection (ATCC, Rockville, MD, USA). The cells were cultured in RPMI-1640 medium or DMEM medium (Thermo Scientific, Rockford, IL, USA) supplemented with $10 \%$ fetal bovine serum and $1 \%$ penicillin/streptomycin (Thermo Scientific). All cells were maintained in a humidified atmosphere of $5 \% \mathrm{CO}_{2}$ at $37^{\circ} \mathrm{C}$.

\section{Small interfering RNA (siRNA) transfection}

For transfection, AGS, MKN28, and MKN74 cells $\left(3 \times 10^{5}\right)$ were seeded in 6-well plates and incubated in a $37^{\circ} \mathrm{C}$ incubator. After 24 hours, the cells were treated with targeted siRNA (LncRNA LUCAT1, $50 \mu \mathrm{M})$ and RNAi negative control $(50 \mu \mathrm{M}$, siCT; Invitrogen, Carlsbad, CA, USA) with Lipofectamine 2000 reagent (Invitrogen) following the manufacturer's protocol. For double knock-out transfection, the LUCAT1 and EZH2 siRNA target sequences were as follows: siLUCAT1_1 sense, CAGAAGAUUCAGAAGAUAAGGAU and antisense, AUCCUUAUCUUCUGACAUCUUCUG; siLUCAT1_2 sense, AGGCCUUGCUCAGUGUCACACAU and antisense, AAUGUGUGACACUGAGCAAGGCCU. siEZH2 sense, UUCAUGCAACACCCAACACU; siEZH2 antisense GAGAGCAGCAGCAAACUCCU.

\section{Construction of LUCAT1 overexpression plasmid}

The LUCAT1 cDNA was amplified by a PCR system (Roche Applied Science, Penzberg, Upper Bavaria, Germany). To insert the cDNA into the pcDNA3.1 (+) expression vector, LUCAT1 NHel_F (acccaagctggctagc CAATGCCCAGACCTCCAG) and LUCAT1_Xbal_R (aaacgggccctctaga TTGACTGCAAGAGCTTGAAG) were used as cloning primers. The pcDNA3.1 (+) expression vector was obtained from Addgene. AGS cells were transfected with $1 \mu \mathrm{g}$ of pcDNA3.1-LUCAT1 for 24 hours using Lipofectamine 2000 (Invitrogen). Transfection was conducted with $1 \mu \mathrm{g}$ of pcDNA3.1-LUCAT1 using Lipofectamine 2000 for 24 hours.

\section{Total RNA extraction and quantitative real-time reverse transcription polymerase chain reaction} Total RNA was obtained from GC tissues and cell lines using TRIzol reagent (Invitrogen). RNA was quantified using Nanodrop (ND-100; Nanodrop Technologies Inc., Wilmington, DE, USA). Purity was determined at a $260 / 280 \mathrm{~nm}$ ratio, and products were loaded on $1 \%$ agarose gel. For cDNA synthesis, 2.0 $\mu \mathrm{g}$ of total RNA was reverse transcribed with SuperscriptTMII (Invitrogen) following the manufacturer's protocol. Relative levels of LUCAT1 were measured by real-time PCR using iQ SYBR Green Supermix (Applied Biosystems Inc., Carlsbad, CA, USA). The Ct value of the sample was normalized to U6 or GAPDH expression, and $2-\Delta \Delta \mathrm{Ct}$ values were calculated. Prim- 
ers used for qRT-PCR are shown in Supplementary Table 1 (only online).

\section{Cell proliferation analysis}

GC cells were transfected with $50 \mu \mathrm{M}$ siLUCAT1s or siCT from 0 to 72 hours. Cell proliferation was performed by CellTiter $96^{\circledR}$ AQueous One Solution Cell Proliferation Assay (MTS assay; Promega, Madison, WI, USA) in 96-well culture plates in timedependent conditions. The plate was allowed to react with MTS reagent for 1 hour in the dark, and products of the reaction were assessed on a spectrophotometric plate reader set at $490 \mathrm{~nm}$ (Multiskan Microplate Photometer; Thermo Scientific). For rescue experiments, cells were transfected with pcDNA-LUCAT1 48 hours after siRNA transfection, and cell proliferation was conducted.

\section{Apoptosis analysis}

AGS, MKN28, and MKN74 cells were transfected with LUCAT1 siRNA or siCT, and the cell pellet was isolated after 48 hours. The cell pellet was resuspended in $1 \times$ binding buffer (BD Bioscience, San Jose, CA, USA) and phosphate-buffered saline (PBS). Cells were stained with propidium iodide and fluorescein isothiocyanate (FITC) annexin V using a FITC-Annexin V kits (BD Bioscience). For analysis by flow cytometry using a FACS verse instrument (BD Biosciences), the stained cells were incubated for 15 minutes. Data were analyzed using Flow Jo software (Treestar, Ashland, OR, USA). Three experiments were conducted for each assay. For apoptosis analysis, the same three GC cell lines were washed with PBS and resuspended in $1 \times$ binding buffer (BD Biosciences). FITC Annexin $\mathrm{V}$ and propidium iodide staining was conducted with the FITC Annexin V detection kit (BD Biosciences) according to the manufacturer's protocol. The ratio of percentage apoptosis was measured by flow cytometry (BD Biosciences).

\section{Scratch wound healing assay and invasion assay}

AGS, MKN28, and MKN74 cells were seeded in 6-well cultured plates transfected with siLUCAT1s or siCT. When the cells achieved approximately $60-80 \%$ confluency, the bottom of the well was scratched equally using a P-20 tip. The width of scratched cells was measured at 0 and $24 \mathrm{~h}$ for AGS and MKN28 cells at 0 and $36 \mathrm{~h}$ for MKN74 cells by microscopy. For invasion assay, two different siLUCATls were transfected into AGS, MKN28, and MKN74 cells, and cells were then reseeded in Matrigel Invasion Chambers (BD Biosciences) in a 24-well culture plate. After $24 \mathrm{~h}$ or $36 \mathrm{~h}$, non-invading cells within the insert chamber were removed, and the upper layer of the trans-well was briefly wiped with a cotton swab. The membrane of the bottom part of the upper chamber was fixed with $5 \%$ acetaldehyde buffer and stained with Crystal violet solution. The invading cells on the membrane were counted under a bright-field microscope.

\section{Soft agar colony formation assay}

To analyze tumorigenicity in vitro, base and top layers of agarose were coated with cells in 96-well plates using CytoSelect ${ }^{\mathrm{TM}}$ 96-Well Cell Transformation Assay (CELL BIOLABS, INC, San Diego, CA, USA). As a base layer, $1.5 \mathrm{~mL}$ of 2X DMEM containing $1 \%$ agarose was poured into each well. After 1 hour of solidification, transfected cells were resuspended in 2X DMEM containing $0.7 \%$ agarose as a top layer and then maintained in an incubator at $37^{\circ} \mathrm{C}$ for $2-3$ weeks. After daily observation, colonies were captured under bright field microscopy.

\section{Western blot}

Cells transfected with siLUCATs or siCT were lysed in IX RIPA buffer (Cell Signaling Technology, Danvers, MA, USA) containing protease inhibitor. Isolated proteins were loaded by 8-15\% SDS-polyacrylamide gel electrophoresis and transferred to a polyvinylidene difluoride membrane (GE Healthcare, Piscataway, NJ, USA). The membrane was blocked for 1 hour at room temperature in tris-phosphate buffer containing $0.1 \%$ Tween 20 with $5 \%$ bovine serum albumin (BD Biosciences) and then incubated with primary antibodies at $4^{\circ} \mathrm{C}$ in a cold room overnight. The following primary antibodies were used for Western blot analysis: epithelial marker E-cadherin (1:1000, BD Biosciences); mesenchymal marker N-cadherin (1:1000, BD Biosciences), Vimentin (1:200, Santa Cruz Biotechnology, Dallas, TX, USA, sc-373717), ZEB1 (1:1000, Cell Signaling Technology, \#3396S), Snail (1:1000, Cell Signaling Technology, \#3879S), PARP (1:1000, Cell Signaling Technology, \#9542), Bcl-xl (1:1000, Cell Signaling Technology, \#2764), Bax (1:1000, Santa Cruz Biotechnology, sc-493), CXXC4 (1:500, Abcam, Cambridge, UK, ab105400), EZH2 (1:1000, Cell Signaling Technology, \#5246S), WNT1 (1:500, Abcam, ab15251), SFRP2 (1:200, Abcam, ab86379), and $\beta$-actin (1:5000, Bioworld Technology, Louis Park, MN, USA, AP0060). Signals were developed in ECL solution (GenDEPOT, Barker, TX, USA) and exposed to an Image Quant LAS 4000 bio-molecular imager for 10 seconds to 6 minutes.

\section{RNA immunoprecipitation (RIP)}

Cells were lysed with immunoprecipitation buffer (Thermo Fisher Scientific, Middlesex County, MA, USA) and resuspended in RIP buffer (Abcam) with mixtures containing RNase inhibitor (GenDEPOT, Hanam, Korea) and protease inhibitor (GenDEPOT). For chromatin shearing, 20 cycles (170-190 W for each cycle) of shearing were performed under cooling conditions with a sonicator. Antibodies were applied to the supernatant and then incubated overnight at $4^{\circ} \mathrm{C}$ with a rotator. After incubation, $20 \mu \mathrm{L}$ of MagnaChip protein magnetic beads (Millipore, Burlington, MA, USA) was added and reacted on a rotator at $4^{\circ} \mathrm{C}$ for 1 hour. After washing twice with RIP buffer, the samples were dissolved with TRIzol reagent or RIPA buffer for further studies. 


\section{Methylation-specific PCR (MS-PCR)}

Genomic DNA was extracted from AGS cells, as well as paired gastric normal and cancer tissues, using a DNeasy Blood \& Tissue kit (Qiagen, Valencia, CA, USA). An EZ DNA methylation-gold kit (Zymo Research, Irvine, CA, USA) was used for DNA bisulfate transformation. Primers were designed for specific methylated and unmethylated sites. The primers used for qRT-PCR are shown in Supplementary Table 2 (only online).

\section{Chromatin immunoprecipitation (ChIp) assay}

Cell lysates were transfected with siCT or siLUCAT1s, followed by chromatin shearing utilizing water bath sonication for 30 cycles under a cooling condition (170-190 W). The fragmented chromatin was isolated with the High-Sensitivity Chip kit (ab185913; Abcam) according to the manufacturer's protocol. Then, $5 \mu \mathrm{g}$ of total chromatin was prepared for ChIp with antiH3K27me3 (ab6002; Abcam) and the mock immunoprecipitation (IgG, ab185913; Abcam) at $4^{\circ} \mathrm{C}$ overnight. After cross- link reversal and DNA purification, qRT-PCR was performed with selected target region primers. The primer sequences for qRT-PCR are shown in Supplementary Table 3 (only online).

\section{Statistical analysis}

All of the analyzed data for continuous variables are presented as a mean \pm standard error. Categorical variables are presented as a number and proportion. Statistical tests included the ttest, $\chi^{2}$ test, Fisher's exact test, and ANOVA test. One-way analysis of variance (ANOVA) was performed with Duncan's multiple range test. The expression of LUCAT1 in GC was classified as low or high based on the average value of LUCAT1 expression. The Kaplan-Meier method and log-rank test were used for survival analysis. A $p$ value $<0.05$ was regarded as a statistically significant difference for group comparisons. All statistical processes were conducted using the statistical software SPSS for Windows (version 18.0; SPSS Inc., Chicago, IL, USA).
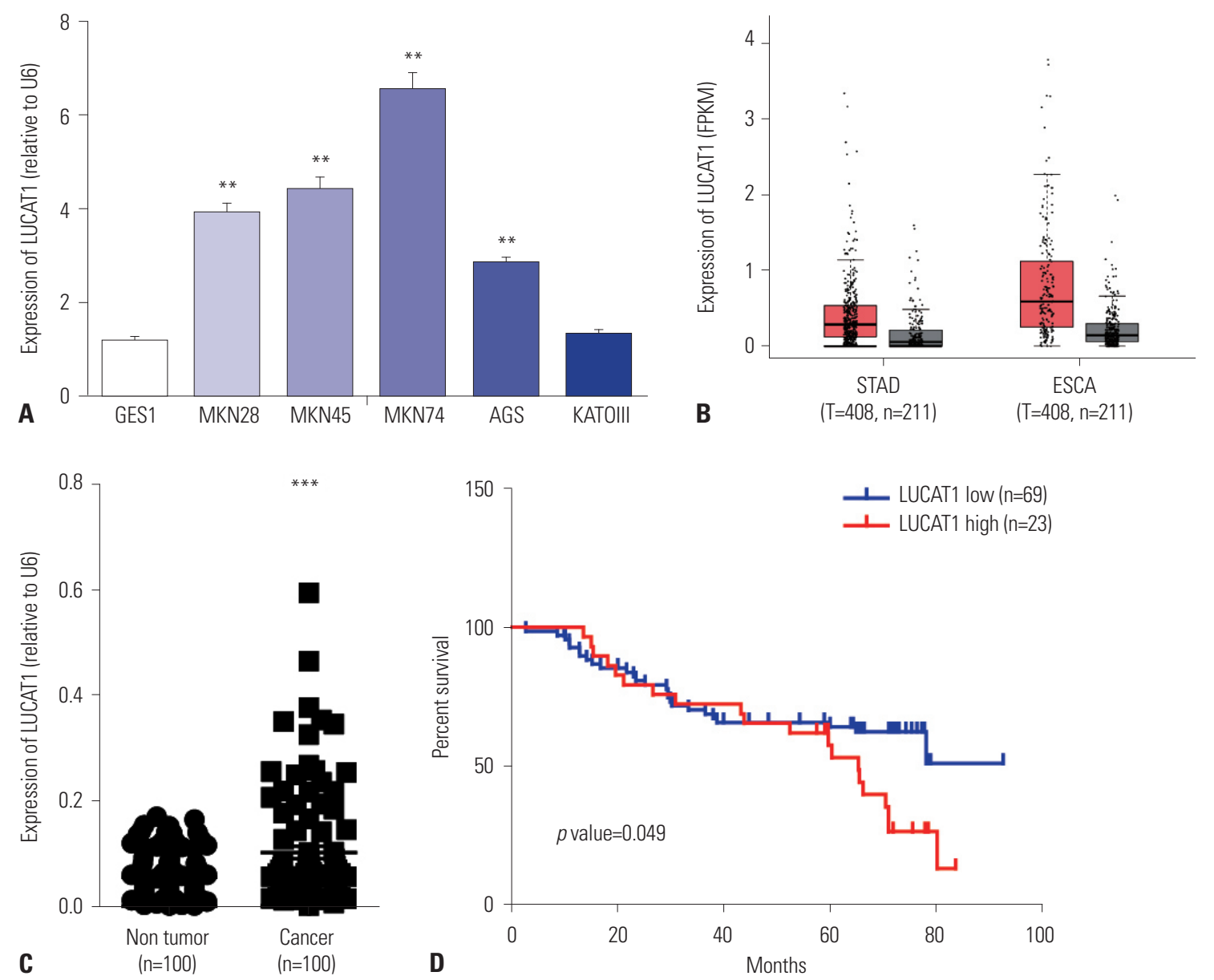

Fig. 1. Expression of LUCAT1 is overexpressed in gastric cancer cell lines and tissues. (A) LUCAT1 expression was upregulated in four gastric cancer cells (AGS, MKN28, MKN45, and MKN74), compared to normal gastric cells (GES1). (B) LUCAT1 expression was determined in stomach adenocarcinoma (STAD) and esophageal adenocarcinoma (ESCA) using GTEx and TCGA data (http://gepia.cancer-pku.cn/detail.php?gene=LUCAT1). (C) LUCAT1 expression was examined in cancer tissues and adjacent non-tumor tissues $(n=100)$. The expression of LUCAT1 was measured by quantitative real-time PCR and calculated by the 2- $\triangle \triangle C T$ method using quantitative real-time PCR. (D) Kaplan-Meier estimates of overall survival in patients with high or low LUCAT1 expression. $p$-values were 0.049 . All of the data are from three independent experiments. Data are presented as the mean \pm s.e.m. Asterisk indicates a statistically significant difference compared to scrambled control. ${ }^{* *} p \leq 0.01$, ${ }^{* * *} p \leq 0.001$. 


\section{RESULTS}

\section{Expression of LUCAT1 in gastric cancer cell lines and tissues}

The expression of LUCAT1 in GC cell lines and gastric tissues was analyzed using qRT-PCR. LUCAT1 was expressed at various levels in five GC cell lines and normal gastric cell line (GES1) (Fig. 1A). Expression of LUCAT1 was higher in MKN28, MKN45, MKN74, and AGS than in normal gastric cells (Fig. 1A). In genotype-tissue expression (GTEx) and Cancer Genome Atlas (TCGA) datasets, LUCAT1 was significantly upregulated in both esophageal adenocarcinoma and GC (STAD) (Fig. 1B). We validated the expression of LUCAT1 in GC specimens of 100 patients who underwent surgery due to GC. Expression of LUCAT1 was significantly higher in GC tissues than in adjacent non-tumor tissues ( $p<0.001)$ (Fig. 1C).

\section{Expression of LUCAT1 and the clinicopathologic characteristics of gastric cancer}

Clinicopathological features of the patients are summarized in Table 1 . The patients' mean age was 65 years, and men accounted for $81 \%$ of all patients. The overall survival rate in the high LUCAT1 expression group tended to be lower than that in the low LUCAT1 expression group ( $p=0.049$ ) (Fig. 1D). When we analyzed disease-free survival in our patients $(p=0.13)$ and the TCGA cohort patients ( $p=0.25)$ based on the expression level of LUCAT1, LUCAT1 did not affect disease-free survival in GC patients (data not shown).

\section{Downregulation of LUCAT1 suppresses the proliferation of gastric cancer cells and induces apoptosis}

For further experiments, we selected AGS, MKN28, and MKN74 cells, which express LUCAT1 at relatively high levels. To evaluate the effects of LUCAT1 on the proliferation of GC cell lines by

Table 1. Relationships between LUCAT1 Expression and Clinicopathological Features in 100 Gastric Cancer Patients

\begin{tabular}{|c|c|c|c|c|}
\hline \multirow{2}{*}{ Clinicopathological variables } & \multirow{2}{*}{ Number in each group } & \multicolumn{2}{|c|}{ LUCAT1 expression $^{\ddagger}$} & \multirow{2}{*}{$p$ value } \\
\hline & & Low (<0.0438), n (\%) & High ( $\geq 0.0438), n(\%)$ & \\
\hline All cases & 100 & 37 & 63 & \\
\hline Age (yr) & & & & 0.037 \\
\hline$<65$ & 56 & $26(70.3)$ & $30(47.6)$ & \\
\hline$\geq 65$ & 44 & $11(29.7)$ & $33(52.4)$ & \\
\hline Sex & & & & 0.522 \\
\hline Male & 62 & $21(56.8)$ & $41(65.1)$ & \\
\hline Female & 38 & $16(43.2)$ & $22(34.9)$ & \\
\hline $\mathrm{HP}$ & & & & 0.449 \\
\hline Positive & 26 & $8(21.6)$ & $18(28.6)$ & \\
\hline Negative & 14 & $5(13.5)$ & $9(14.3)$ & \\
\hline Location* & & & & 0.225 \\
\hline Upper third & 12 & $5(13.5)$ & $7(11.1)$ & \\
\hline Middle third & 38 & $17(45.9)$ & $21(33.3)$ & \\
\hline Lower third & 50 & $15(40.5)$ & $35(55.6)$ & \\
\hline T stage & & & & 0.380 \\
\hline T1 & 16 & $4(10.8)$ & $12(19)$ & \\
\hline $\mathrm{T} 2$ & 8 & $4(10.8)$ & $4(6.3)$ & \\
\hline T3 & 27 & $9(24.3)$ & $18(28.6)$ & \\
\hline $\mathrm{T} 4$ & 49 & $20(54.1)$ & $29(46)$ & \\
\hline Depth of tumor invasion & & & & 0.810 \\
\hline $\mathrm{T} 1 / \mathrm{T} 2$ & 24 & $8(21.6)$ & $16(25.4)$ & \\
\hline $\mathrm{T} 3 / \mathrm{T} 4$ & 76 & $29(78.4)$ & $47(74.6)$ & \\
\hline Lymph node metastasis & & & & 0.167 \\
\hline Absent & 28 & $7(18.9)$ & 21 (33.3) & \\
\hline Present & 72 & $30(81.1)$ & $42(66.7)$ & \\
\hline N stage & & & & 0.355 \\
\hline NO & 28 & $7(18.9)$ & 21 (33.3) & \\
\hline N1 & 17 & $9(24.3)$ & $8(12.7)$ & \\
\hline N2 & 26 & $9(24.3)$ & $17(27.0)$ & \\
\hline N3 & 29 & $12(32.4)$ & $17(27.0)$ & \\
\hline
\end{tabular}


Table 1. Relationships between LUCAT1 Expression and Clinicopathological Features in 100 Gastric Cancer Patients (continued)

\begin{tabular}{|c|c|c|c|c|}
\hline \multirow{2}{*}{ Clinicopathological variables } & \multirow{2}{*}{ Number in each group } & \multicolumn{2}{|c|}{ LUCAT1 expression ${ }^{\ddagger}$} & \multirow{2}{*}{$p$ value } \\
\hline & & Low (<0.0438), n (\%) & High $(\geq 0.0438), n(\%)$ & \\
\hline Lymphovascular invasion & & & & 0.383 \\
\hline Absent & 33 & $10(27.0)$ & $23(36.5)$ & \\
\hline Present & 67 & $27(73.0)$ & $40(63.5)$ & \\
\hline Stage $^{\dagger}$ & & & & 0.837 \\
\hline I, II & 42 & $15(40.5)$ & $27(42.9)$ & \\
\hline III & 58 & $22(59.5)$ & $36(57.1)$ & \\
\hline Lauren's classification & & & & 0.175 \\
\hline Intestinal & 49 & $15(40.5)$ & $34(54)$ & \\
\hline Diffuse & 43 & $18(48.6)$ & $25(39.7)$ & \\
\hline Mixed & 8 & $4(10.8)$ & $4(6.3)$ & \\
\hline Differentiation (Diff. vs. Undiff.) & & & & 0.532 \\
\hline Well to moderate & 42 & $14(37.8)$ & $28(45.2)$ & \\
\hline PD to SRC & 57 & $23(62.2)$ & $34(54.8)$ & \\
\hline Serum CEA value & & & & 0.799 \\
\hline$\leq 5$ & 80 & $29(78.4)$ & $51(81.0)$ & \\
\hline$>5$ & 20 & $8(21.6)$ & $12(19.0)$ & \\
\hline Serum CA19-9 value & & & & 0.667 \\
\hline$\leq 37$ & 94 & 34 (91.9) & $60(95.2)$ & \\
\hline$>37$ & 6 & $3(8.1)$ & $3(4.8)$ & \\
\hline Preop chemotherapy & & & & 1.000 \\
\hline No & 96 & 36 (97.3) & $60(95.2)$ & \\
\hline Yes & 4 & $1(2.7)$ & $3(4.8)$ & \\
\hline Adjuvant chemotherapy & & & & 0.059 \\
\hline No & 43 & $11(29.7)$ & $32(50.8)$ & \\
\hline Yes & 57 & $26(70.3)$ & 31 (49.2) & \\
\hline
\end{tabular}

HP, Helicobacter pylori; PD, poorly differentiated adenocarcinoma; SRC, signet ring cell carcinoma; CEA, carcinoembryonic antigen; CA19-9, carbohydrate antigen 19-9.

* Location was classified according to the American Joint Cancer Committee on Cancer staging system, 'Stage was classified according to the 7th edition of the

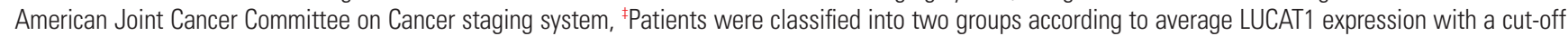
value of 0.0488 .

MTS assay, GC cells were incubated with siRNAs for LUCAT1 and pcDNA-LUCAT1. After treatment of AGS, MKN28, and MKN74 cells with $50 \mu \mathrm{M}$ siLUCAT1, there was about a 50\% decline in LUCAT1 expression, compared to cells transfected with control siRNA (siCT) (Fig. 2A). Treatment of cells with $100 \mu \mathrm{M}$ siLUCAT1 did not result in a substantially greater decline in LUCAT1, compared to using $50 \mu \mathrm{M}$ siLUCAT1, in AGS and MKN 74 cells. Therefore, further experiments were conducted at an siLUCAT1 concentration of $50 \mu \mathrm{M}$. pcDNA-LUCAT1 induced overexpression of LUCAT1 by about 40-fold, compared to pcDNA vector alone, in three different GC cell lines (Fig. 2B). Following transfection of siRNAs, transfection with both siLUCAT1_1 and siLUCAT1_2 significantly decreased AGS, MKN28, and MKN74 cell viability, compared to siCT. Restoration of LUCAT1 expression by pcDNA-LUCAT1 transfection in siLUCAT1-transfected cells significantly rescued cell proliferation in these cells (Fig. 2C).

Next, we performed PI/Annexin V staining and flow cytometry to evaluate the incidence of apoptotic cell death after al- teration of LUCAT1. AGS, MKN28, and MKN74 cells transfected with siLUCAT1s showed a significant increase in early-tolate apoptotic ratio, compared to cells transfected with siCT (Fig. 2D). Poly (ADP-ribose) polymerase was cleaved by transfection of siLUCAT1 in AGS, MKN28, and MKN74 cells (Fig. 2E). siLUCATs increased the expression of Bax and suppressed the expression of Bcl-xl in three different cell lines (Fig. 2E). These changes were reversed by overexpression LUCAT1 upon transfection of pcDNA-LUCAT1.

\section{siLUCAT1 suppresses invasion and migration in gastric cancer cells}

Transfection of AGS, MKN28, and MKN74 cells with two different LUCAT1s significantly reduced invasion ability in Matrigel invasion assay (Fig. 3A). In wound-healing assay, siLUCAT1s repressed the migration ability of three GC cell lines, compared with siCT, which was consistent with the invasion assay results (Fig. 3B). These reductions in invasion and migration were reversed by overexpression of LUCATl upon trans- 
fection with pcDNA-LUCAT1 (Fig. 3A and B).

Next, we performed soft agar colony formation assay to assess in vitro cellular anchorage-independent growth. The number and size of forming colonies declined notably in AGS cells transfected with siLUCAT1s, compared to cells transfected with siCT (Fig. 3C). Next, we evaluated differential expres-
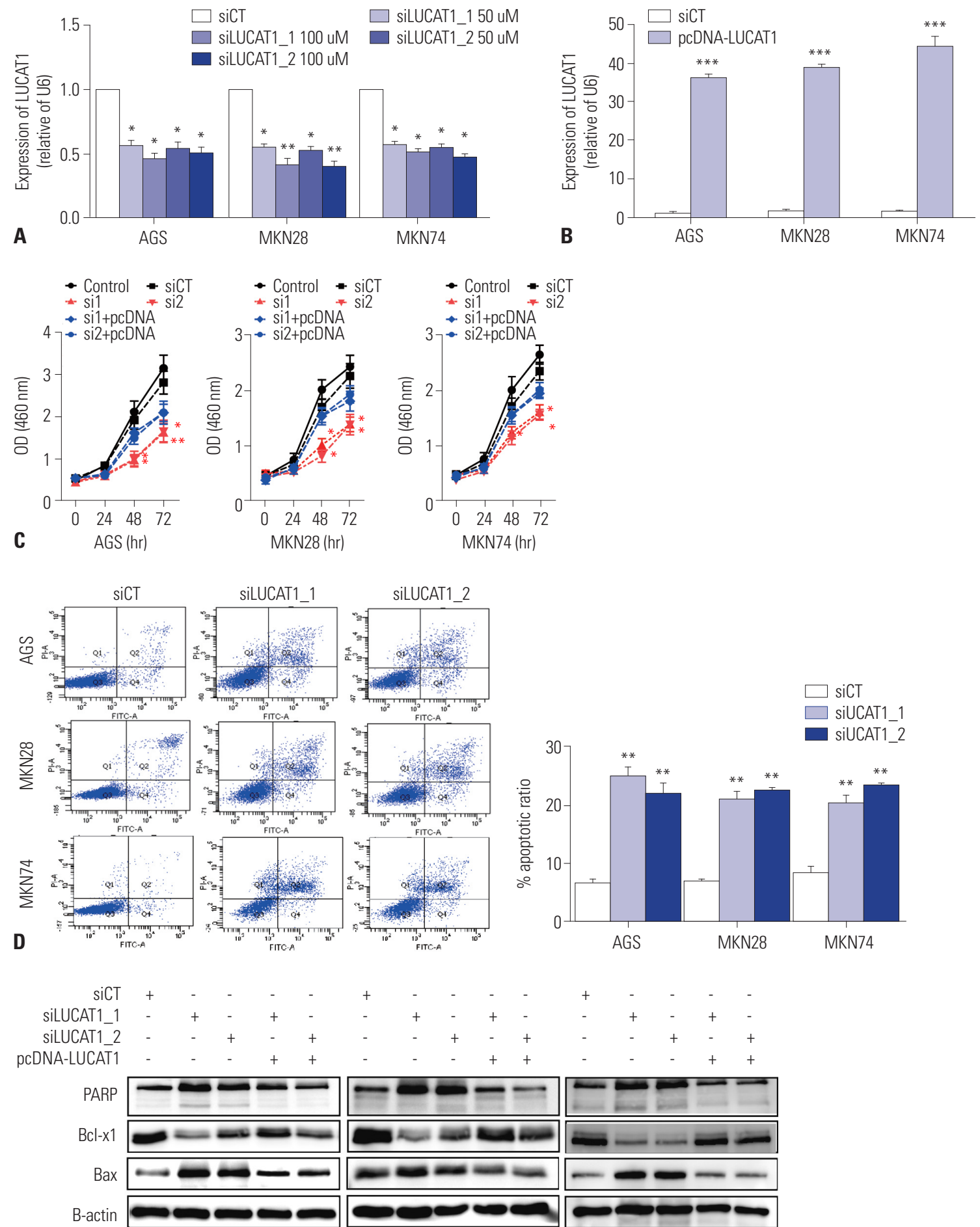

E

AGS

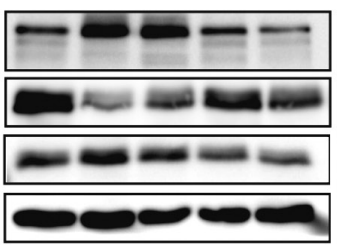

MKN28

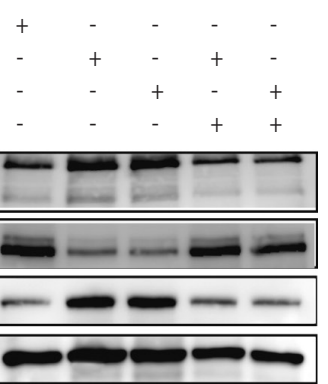

MKN74

Fig. 2. Knockdown of LUCAT1 expression inhibits cell proliferation and induces apoptosis in gastric cancer cells. Expression of LUCAT1 was measured by qRT-PCR in AGS, MKN28, and MKN74 cells transfected with (A) siRNAs and (B) overexpression of LUCAT1 (pcDNA-LUCAT1). (C) Cell proliferation was detected by MTS assay. (D) AGS, MKN28, and MKN74 cells were transfected with siCT or siLUCAT1s, followed by apoptosis assay using $\mathrm{PI} /$ Annexin-V staining. (E) Apoptotic markers were detected in transfected AGS, MKN28, and MKN74 cells. All of the data are from three independent experiments. Data are presented as the mean \pm s.e.m. Asterisk indicates a statistically significant difference compared to scrambled control. * $p \leq 0.05$, ${ }^{* *} p \leq 0.01,{ }^{* * *} p \leq 0.001$. 

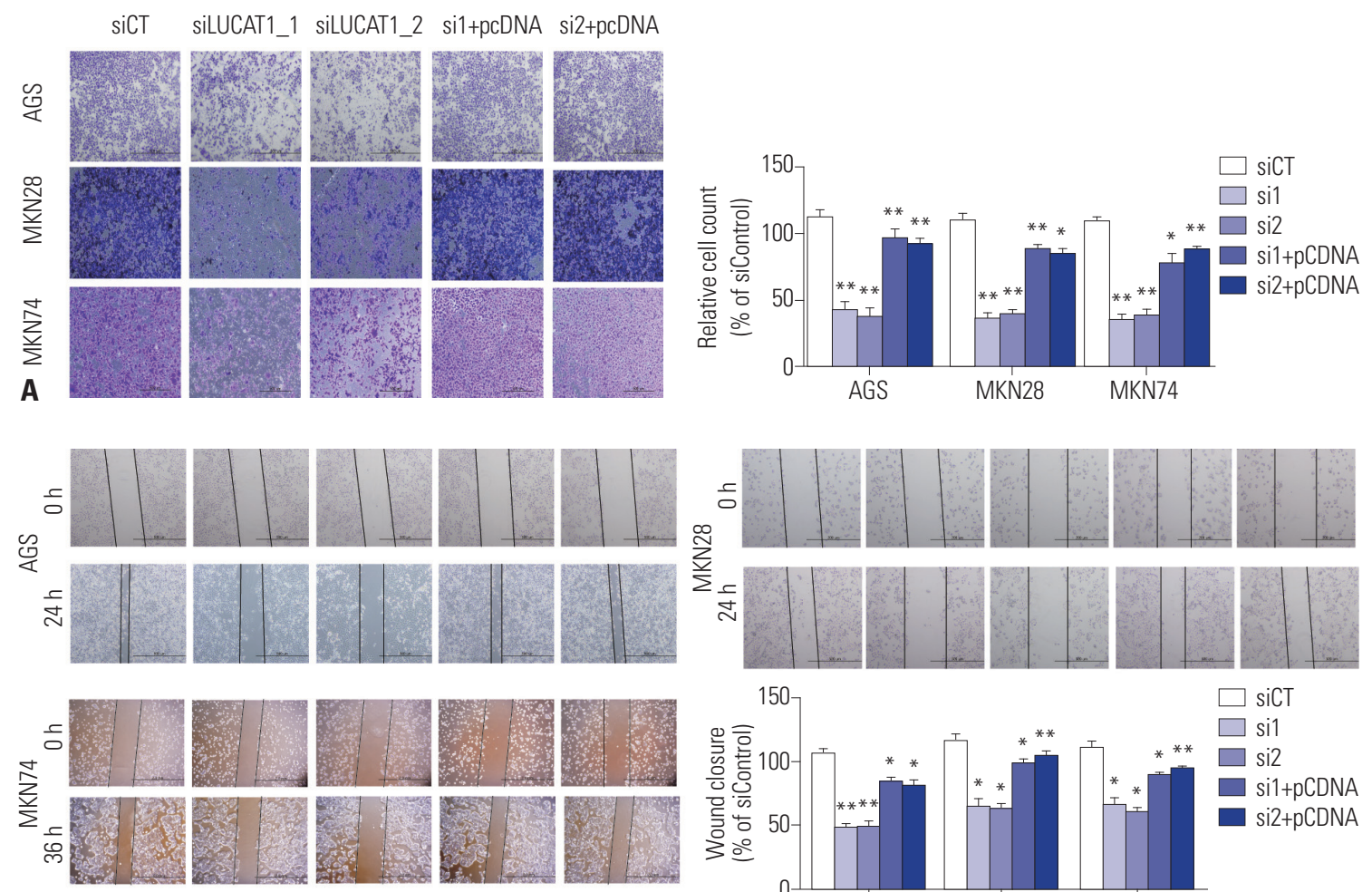

B
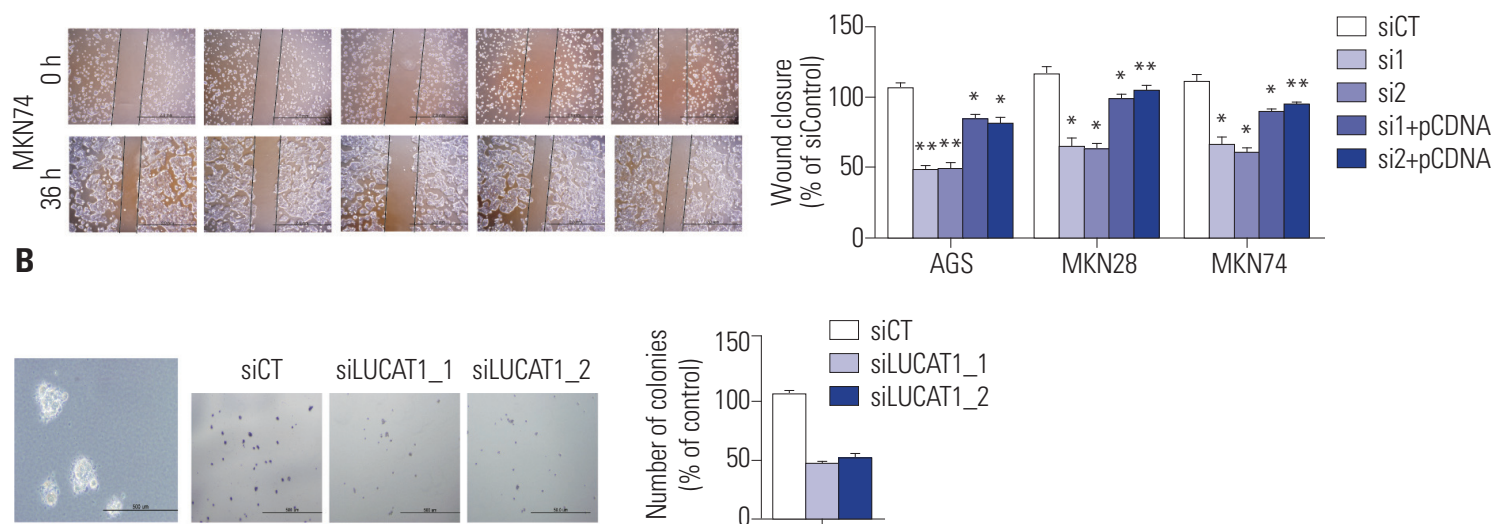

C

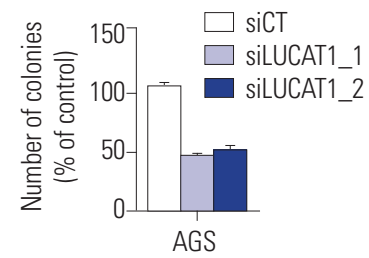

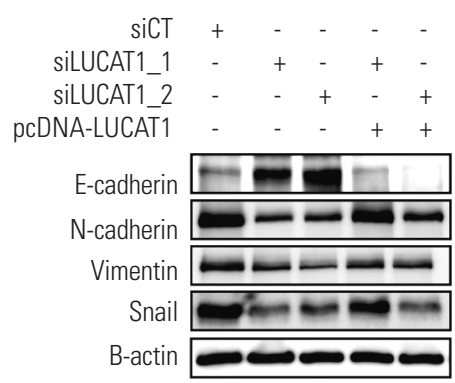

AGS

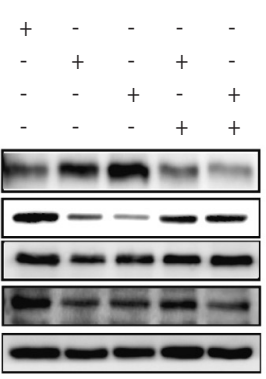

MKN28

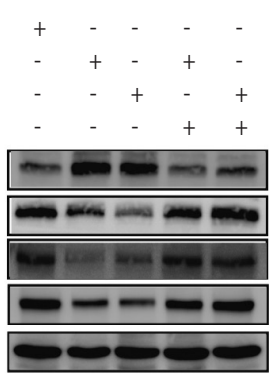

MKN74

Fig. 3. siLUCAT1s suppress the cell migration and invasion of AGS, MKN28, and MKN74 cells. (A) Invasion assays and (B) wound healing assays were performed, followed by siCT or siLUCAT1s. At 48 hours after transfection of siLUCAT1s, pcDNA-LUCAT1 was transfected into cells, and then migration and invasion was observed by microscopy. Bar graph shows the percentage of wound closures and the number of invading cells. (C) Soft agar colony formation was carried out during the knockdown of LUCAT1 in AGS cells. (D) EMT markers were performed by immunoblotting transfected AGS, MKN28, and MKN74 cells. All of the data are from three independent experiments. Data are presented as the mean \pm s.e.m. Asterisk indicates a statistically significant difference compared to scrambled control. ${ }^{*} p \leq 0.05,{ }^{* *} p \leq 0.01$.

sion of EMT markers using Western blot. The expression of the mesenchymal markers Snail, N-cadherin, and vimentin were reduced, whereas that of E-cadherin was increased after transfection of cells with siLUCATls, compared to siCT, although these were revered by transfection of pcDNA-LUCAT1 (Fig. 3D). Taken together, these finding indicated that LUCAT1 affects cell migration and invasion, which are involved in car- cinogenesis.

\section{LUCAT1 regulates methylation of $C X X C 4$ and SFRP2 through the EZH2/Wnt/ $\beta$-catenin pathway}

In our previous study, microarray analysis revealed 770 cancerrelated genes in ESCC after transfection with siLUCAT $1 .{ }^{27}$ Using these microarray data, we performed new analysis focus- 
ing on tumor suppressor genes that have already been reported to be reduced due to methylation in GC. In doing so, we confirmed that mRNA expression of tumor suppressor genes, such as DKK3, PCDH10, ZNF331, CXXC4, and SFRP2, were increased by siLUCAT1 more than twice that of siCT (data not shown), and we decided to analyze CXXC4 and SFRP2 for fur- ther experiments: $C X X C 4$ and SFRP2 have been reported to be downstream targets of EZH2/Wnt/ $\beta$-catenin in GC. ${ }^{34,35}$ We assessed the mRNA levels and methylation status of the candidate tumor suppressor genes, CXXC4 and SFRP2, and found that the methylation status of these two genes was significantly elevated by siLUCAT1 transfection, compared with siCT
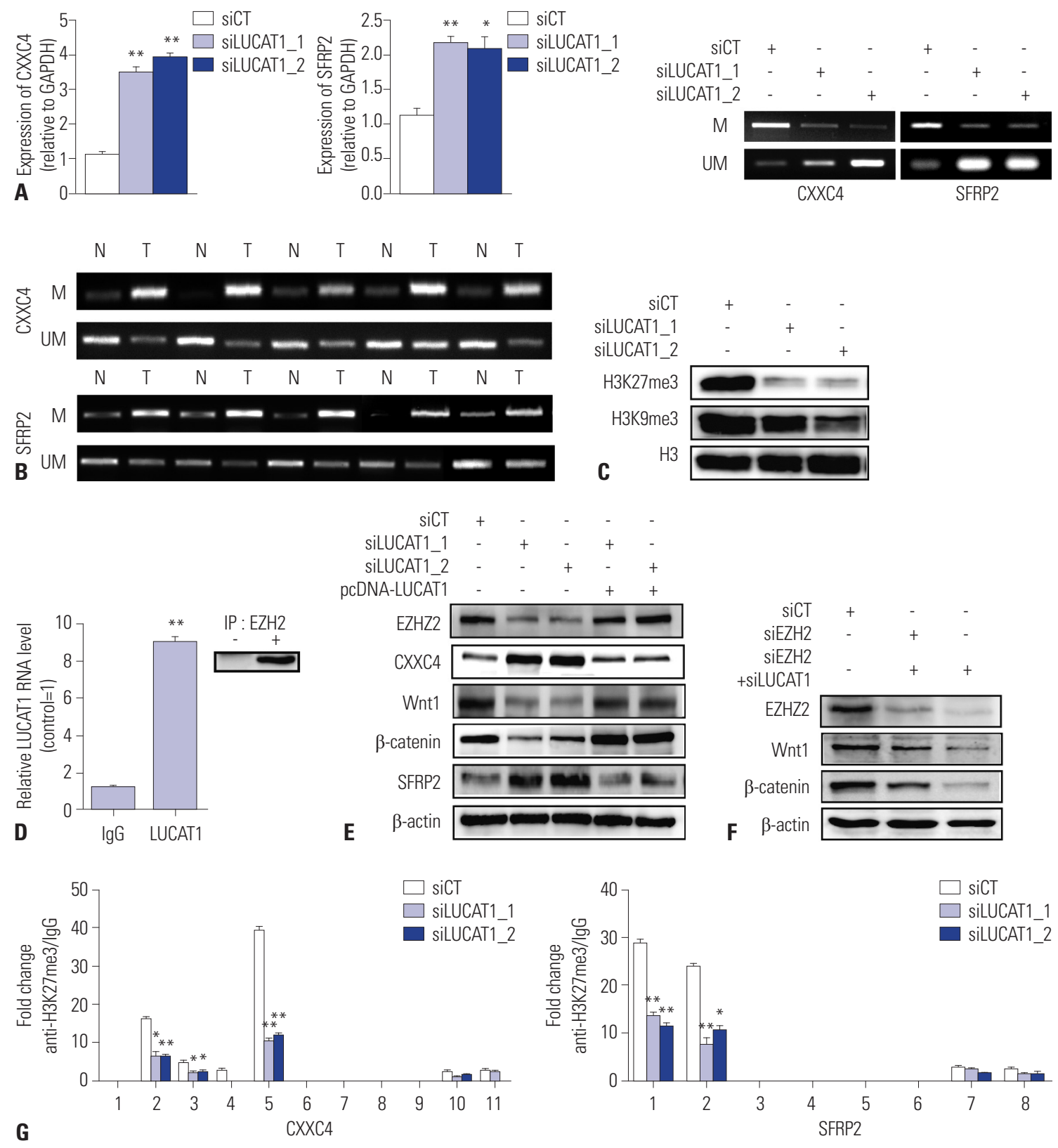

Fig. 4. LUCAT1 controls the methylation of CXXC4 and SFRP2through interactions with EZH2 and H3K27me3. (A) The expression of CXXC4 and SFRP2 was detected by qRT-PCR and MS-PCR in AGS cells treated with siCT or siLUCAT1s. (B) The methylation status of CXXC4 and SFRP2 was estimated in five cancer tissues and adjacent non-tumor tissues via MS-PCR. (C) After treatment with siCT or siLUCAT1s, H3K27me3 and H3K9me3 antigens were detected by immunoblotting. (D) AGS cell lysates were immunoprecipitated with anti-EZH2 antibody, and the interaction between EZH2 and LUCAT1 was confirmed through RIP analysis. (E) The translational levels of EZH2 and its downstream signals, Wnt and $\beta$-catenin, were measured by immunoblotting. (F) Immunoblotting was performed using AGS cells transfected with siCT or siEZH2 and with both siEZH2 and siLUCAT1 (double-knockdown). (G) H3K27me3 enrichment on CXXC4 and SFRP2 genes in ChIP assay in siCT- or siLUCAT1s-transfected AGS cells by qRT-PCR at respective potential regulatory regions. All of the data are from three independent experiments. Data are presented as the mean \pm s.e.m. Asterisk indicates a statistically significant difference compared to scrambled control. ${ }^{*} p \leq 0.05,{ }^{* *} p \leq 0.01$. 
transfection, in AGS cells (Fig. 4A). This was confirmed in five paired adjacent non-tumor tissues and cancer tissues by MSPCR (Fig. 4B). Therefore, our findings confirmed that LUCAT1 is involved in the methylation of tumor suppressor genes in GC. The expressions of $H 3 \mathrm{~K} 9 \mathrm{me} 3$ and $H 3 \mathrm{~K} 27 \mathrm{me} 3$ were reduced by treatment with siLUCAT1s, thus this reduction of histone markers was correlated with methylation of tumor suppressor genes (Fig. 4C).

Next, we analyzed the effects of LUCAT1 on EZH2, which is the major chromatin repressive modification marker that interacts with H3K27me3. LUCAT1 and EZH2 interaction was established by RIP assay; LUCAT1 was abundant in the antiEZH2 RIP fraction, compared to IgG fraction (Fig. 4D). EZH2, acting as an upstream regulator of Wnt/ $\beta$-catenin, could suppress WNT expression and induce $H 3 K 27$ methylation, leading to inactivation of the $\mathrm{Wnt} / \beta$-catenin pathway ${ }^{36}$; however, no previous association with LUCAT1 has been identified. To confirm the relationship between LUCAT1 and EZH2/Wnt/ $\beta$-catenin, we performed Western blot assay and found that the protein levels of EZH2, Wnt, and $\beta$-catenin were significantly increased after LUCAT1 knockdown, while overexpression of LUCAT1 almost fully reversed the changes by LUCAT1 knockdown (Fig. 4E). The expressions of CXXC4 and SFRP2, which are targets of EZH2/Wnt/ $\beta$-catenin, also changed under the same conditions (Fig. 4E). Next, we introduced siEZH2 and siLUCAT1 into cells to perform a double-knockout of EZH2 and LUCAT1. Double knockdown of EZH2 and LUCAT1 further decreased levels of EZH2, Wnt, and $\beta$-catenin, compared to knockdown of EZH2 or LUCAT1 alone (Fig. 4F).

To confirm interactions among H3K27me3, LUCAT1, CXXX4, and $S F R P 2$, we performed ChIP assays to assess signals of H3K27me3 in CXXC4 and SFRP2 genomic regions according to the presence or absence of LUCAT1, and found a significant decline in H3K27me3 signals at specific sites in the genes (indicated in primers 2,5 for CXXC4 and primers 1,2 for SFRP2) upon knockdown of LUCAT1 cells (Fig. 4G). This result indicated that LUCAT1 is involved in the binding of H3K27me3 to CXXC4 and SFRP2 genes.

\section{DISCUSSION}

Among the various mechanisms involved in the development and progression of cancer, IncRNA has recently been shown to play a significant role therein. We previously reported that LUCAT1 promotes ESCC by inhibiting the expression of tumor suppressor genes. Specifically, we found that LUCAT1 regulates the stability of DNMT1, which is likely to result in broad alterations in DNA methylation in various cancers. ${ }^{27}$ In the present study, LUCAT1 was upregulated in GC, compared to adjacent non-tumor tissue. Transfecting GC cells with siRNAs against LUCAT1 reduced the proliferation, migration, invasion, and the colony formation ability of the GC cells. Transfection of cells with pcDNA-LUCAT1 rescued this inhibition. Knockdown of LUCAT1 reduced the methylation status of $C X X C 4$ and SFRP2 and the expression of H3K27me3 by targeting EZH2, which leads to activation of the Wnt/ $\beta$-catenin signaling pathway. Moreover, silencing of LUCAT1 inhibited the binding of EZH2 to CXXC4, H3K27me3, and SFRP2. Our results indicate that LUCAT1 is involved in epigenetic repression of tumor suppressor genes in GC.

Since LUCAT1 was first introduced in 2013 under the name of smoke and cancer-associated lncRNA-1 (SCAL1), it has been shown to be expressed in serous ovarian cancer, clear cell renal cell carcinoma, colorectal cancer, bladder cancer, osteosarcoma, glioma, esophageal, head and neck squamous cell carcinoma, prostate, hepatocellular carcinoma, triple negative breast cancer, and cervical cancer. Chi, et al. ${ }^{37}$ reported that LUCAT1 promotes GC by regulating the miR-134-5p/YWHAZ axis. Here, we found that LUCAT1 expression was higher in GC tissue than in adjacent non-tumor tissue. When we simply compared the relative increases in LUCAT1 in GC and ESCC in relation to overall survival in our current and previous studies, we inferred that LUCAT1 may be a better target for squamous cell carcinoma than adenocarcinoma due to the large increase in LUCAT1 expression in ESCC, compared to GC. To confirm this, however, additional GC and ESCC cohorts are needed, and we are now doing this project.

LUCAT1 has been shown to be involved in GC carcinogenesis via methylated tumor suppressor genes, such as $C X X C 4$ and SFPR2. CXXC4 was reported to be a novel tumor suppressor in GC. ${ }^{34,38}$ CXXC4 acts as a negative regulator of the Wnt signaling pathway and contributes to tumor suppression. Alteration of the methylation status of $C X X C 4$ or mutation of this gene have been reported in GC. ${ }^{37}$ We examined the methylation status of CXXC4 and SFRP2 in non-tumor and cancer tissues from five patients, and found that these two genes were more highly methylated in cancer tissue than in non-tumor tissue. We also found that siLUCAT1 reduced methylation of CXXC4 and SFRP2 in GC cells.

EZH2 has been reported to induce aberrant activation of WNT signaling by downregulating CXXC4 expression in GC. ${ }^{34}$ Additionally, recent studies have shown that IncRNA can impact methylation status by binding with EZH2 in various cancers. $^{39,40}$ siLUCAT1 reduced the expression of EZH2 and induced the expression of CXXC4, while overexpression of LUCAT1 restored the expression levels of EZH2 and CXXC4 in Western blot experiments. This suggests that LUCAT1 regulates the expression of E-cadherin, N-cadherin, and vimentin, which are all markers of WNT and EMT signaling. The expression of $\mathrm{Wnt} / \beta$-catenin was more prominently reduced when siEZH2 and siLUCAT1 were administered simultaneously, compared to when only siEZH2 was administered. These results suggest that, in addition to EZH2, LUCAT1 is involved in Wnt/ $\beta$-catenin signaling, and this should be further investigated.

In this study, we demonstrated that EZH2 directly binds 
with LUCAT1 in RIP assay, and the mechanism by which LUCAT1 enhances EZH2 can be predicted from our previous experiments. ${ }^{27}$ Our results indicated that LUCAT1 regulates the ubiquitination of DNMT1 mediated by the E3 ubiquitin-protein ligase UHRF1. Accordingly, decreasing protein levels of EZH2 by siLUCAT1 in our present result is expected to be a function of ubiquitination in EZH2. Additional studies should be performed to discover the relationship between EZH2 and LUCAT1.

Furthermore, siLUCAT1 reduced the expression of H3K27me3 in GC cell lines. In ChIP analysis, siLUCAT1 selectively inhibited the binding of $\mathrm{H} 3 \mathrm{~K} 27 \mathrm{me} 3$ to $\mathrm{CpG}$ islands of $\mathrm{CXXC4}$ and SFRP2 genes. This implies that LUCAT1 is involved in the EZH2-mediated repression of H3K27me3, as well as in methylation of the tumor suppressor genes CXXC4 and SFRP2.

In conclusion, LUCAT1 inhibits $C X X C 4$ expression through induction of EZH2 expression, H3K27me3-mediated epigenetic regulation, and methylation of CXXC4 and SFRP2. LUCAT1 can thereby activate the WNT pathway and play a role in GC development. Our study proposes a new mechanism by which LUCAT1 interacts with a key pathway in GC development.

\section{ACKNOWLEDGEMENTS}

This research was supported by the Brain Korea 21 PLUS Project for Medical Science, Yonsei University.

This study was financially supported by the "Kiturami" Faculty Research Assistance Program of Yonsei University College of Medicine for 2011 (6-2011-0169), as well as a National Research Foundation of Korea (NRF) grant funded by the South Korean government (MSIT) (2020R1A2B5B0100204711).

\section{AUTHOR CONTRIBUTIONS}

Conceptualization: all authors. Data curation: Hyo Joo Byun. Formal analysis: Hyo Joo Byun. Funding acquisition: Sang Kil Lee. Investigation: Jung-Ho Yoon. Methodology: Hyo Joo Byun and Jung-Ho Yoon. Project administration: Sang Kil Lee. Resources: Jung-Ho Yoon and Sang Kil Lee. Software: Hyo Joo Byun. Supervision: Sang Kil Lee. Validation: Hyo Joo Byun. Visualization: Hyo Joo Byun. Writing-original draft: Hyo Joo Byun. Writing-review \& editing: Jung-Ho Yoon and Sang Kil Lee. Approval of final manuscript: all authors.

\section{ORCID iDs}

Hyo Joo Byun Jung-Ho Yoon https://orcid.org/0000-0002-7039-9534 Sang Kil Lee https://orcid.org/0000-0002-9188-896X https://orcid.org/0000-0002-0721-0364

\section{REFERENCES}

1. Cancer Genome Atlas Research Network. Comprehensive molecular characterization of gastric adenocarcinoma. Nature 2014; 513:202-9.

2. Herszényi L, Tulassay Z. Epidemiology of gastrointestinal and liv- er tumors. Eur Rev Med Pharmacol Sci 2010;14:249-58.

3. Maruyama R, Suzuki H, Yamamoto E, Imai K, Shinomura Y. Emerging links between epigenetic alterations and dysregulation of noncoding RNAs in cancer. Tumour Biol 2012;33:277-85.

4. Morlando M, Fatica A. Alteration of epigenetic regulation by long noncoding RNAs in cancer. Int J Mol Sci 2018;19:570.

5. Li T, Mo X, Fu L, Xiao B, Guo J. Molecular mechanisms of long noncoding RNAs on gastric cancer. Oncotarget 2016;7:8601-12.

6. Jones PA, Baylin SB. The fundamental role of epigenetic events in cancer. Nat Rev Genet 2002;3:415-28.

7. Jones PA, Baylin SB. The epigenomics of cancer. Cell 2007;128: 683-92.

8. Chiurillo MA. Role of the Wnt/ $\beta$-catenin pathway in gastric cancer: an in-depth literature review. World J Exp Med 2015;5:84-102.

9. Kojima T, Shimazui T, Hinotsu S, Joraku A, Oikawa T, Kawai K, et al. Decreased expression of CXXC4 promotes a malignant phenotype in renal cell carcinoma by activating Wnt signaling. Oncogene 2009;28:297-305

10. Su M, Xiao Y, Tang J, Wu J, Ma J, Tian B, et al. Role of lncRNA and EZH2 interaction/regulatory network in lung cancer. J Cancer 2018; 9:4156-65.

11. Lu Y, Zhao X, Liu Q, Li C, Graves-Deal R, Cao Z, et al. lncRNA MIR100HG-derived miR-100 and miR-125b mediate cetuximab resistance via Wnt/ $\beta$-catenin signaling. Nat Med 2017;23:1331-41.

12. You BH, Yoon JH, Kang H, Lee EK, Lee SK, Nam JW. HERES, a lncRNA that regulates canonical and noncanonical Wnt signaling pathways via interaction with EZH2. Proc Natl Acad Sci U S A 2019; 116:24620-9.

13. Hu XY, Hou PF, Li TT, Quan HY, Li ML, Lin T, et al. The roles of Wnt/ $\beta$-catenin signaling pathway related lncRNAs in cancer. Int J Biol Sci 2018;14:2003-11.

14. Zhou W, He X, Chen Z, Fan D, Wang Y, Feng H, et al. LncRNA HOTAIR-mediated Wnt/ $\beta$-catenin network modeling to predict and validate therapeutic targets for cartilage damage. BMC Bioinformatics 2019;20:412.

15. Li YF, Zhang J, Yu L. Circular RNAs regulate cancer onset and progression via $W n t / \beta$-catenin signaling pathway. Yonsei Med J 2019; 60:1117-28

16. Ponting CP, Oliver PL, Reik W. Evolution and functions of long noncoding RNAs. Cell 2009;136:629-41.

17. Esteller M. Non-coding RNAs in human disease. Nat Rev Genet 2011;12:861-74.

18. Nana-Sinkam SP, Croce CM. Non-coding RNAs in cancer initiation and progression and as novel biomarkers. Mol Oncol 2011; 5:483-91.

19. Zhou Z, Lin Z, Pang X, Tariq MA, Ao X, Li P, et al. Epigenetic regulation of long non-coding RNAs in gastric cancer. Oncotarget 2017; 9:19443-58.

20. Guil S, Esteller M. RNA-RNA interactions in gene regulation: the coding and noncoding players. Trends Biochem Sci 2015;40:24856.

21. Huarte M. The emerging role of lncRNAs in cancer. Nat Med 2015; 21:1253-61

22. Mercer TR, Dinger ME, Mattick JS. Long non-coding RNAs: insights into functions. Nat Rev Genet 2009;10:155-9.

23. Youn $\mathrm{YH}$, Byun HJ, Yoon JH, Park CH, Lee SK. Long noncoding RNA N-BLR upregulates the migration and invasion of gastric adenocarcinoma. Gut Liver 2019;13:421-9.

24. Feng $\mathrm{H}$, Liu X. Interaction between ACOT7 and LncRNA NMRAL2P via methylation regulates gastric cancer progression. Yonsei Med J 2020;61:471-81.

25. Zhou H, Sun L, Wan F. Molecular mechanisms of TUG1 in the proliferation, apoptosis, migration and invasion of cancer cells. Oncol 
Lett 2019;18:4393-402.

26. Di W, Weinan X, Xin L, Zhiwei Y, Xinyue G, Jinxue T, et al. Long noncoding RNA SNHG14 facilitates colorectal cancer metastasis through targeting EZH2-regulated EPHA7. Cell Death Dis 2019;10: 514.

27. Yoon JH, You BH, Park CH, Kim YJ, Nam JW, Lee SK. The long noncoding RNA LUCAT1 promotes tumorigenesis by controlling ubiquitination and stability of DNA methyltransferase 1 in esophageal squamous cell carcinoma. Cancer Lett 2018;417:47-57.

28. Zheng Z, Zhao F, Zhu D, Han J, Chen H, Cai Y, et al. Long non-coding RNA LUCAT1 promotes proliferation and invasion in clear cell renal cell carcinoma through AKT/GSK-3 $\beta$ signaling pathway. Cell Physiol Biochem 2018;48:891-904.

29. Thai P, Statt S, Chen CH, Liang E, Campbell C, Wu R. Characterization of a novel long noncoding RNA, SCAL1, induced by cigarette smoke and elevated in lung cancer cell lines. Am J Respir Cell Mol Biol 2013;49:204-11.

30. Sun Y, Jin SD, Zhu Q, Han L, Feng J, Lu XY, et al. Long non-coding RNA LUCAT1 is associated with poor prognosis in human nonsmall lung cancer and regulates cell proliferation via epigenetically repressing p21 and p57 expression. Oncotarget 2017;8:28297-311.

31. Zhou Q, Hou Z, Zuo S, Zhou X, Feng Y, Sun Y, et al. LUCAT1 promotes colorectal cancer tumorigenesis by targeting the ribosomal protein L40-MDM2-p53 pathway through binding with UBA52. Cancer Sci 2019;110:1194-207.

32. Gao YS, Liu XZ, Zhang YG, Liu XJ, Li LZ. Knockdown of long noncoding RNA LUCAT1 inhibits cell viability and invasion by regu- lating miR-375 in glioma. Oncol Res 2018;26:307-13.

33. Han Z, Shi L. Long non-coding RNA LUCAT1 modulates methotrexate resistance in osteosarcoma via miR-200c/ABCB1 axis. Biochem Biophys Res Commun 2018;495:947-53.

34. Lu H, Sun J, Wang F, Feng L, Ma Y, Shen Q, et al. Enhancer of zeste homolog 2 activates wnt signaling through downregulating CXXC finger protein 4. Cell Death Dis 2013;4:e776.

35. Bracken AP, Dietrich N, Pasini D, Hansen KH, Helin K. Genomewide mapping of Polycomb target genes unravels their roles in cell fate transitions. Genes Dev 2006;20:1123-36.

36. Wang H, Meng Y, Cui Q, Qin F, Yang H, Chen Y, et al. MiR-101 targets the EZH2/Wnt/ $\beta$-catenin the pathway to promote the osteogenic differentiation of human bone marrow-derived mesenchymal stem cells. Sci Rep 2016;6:36988.

37. Chi J, Liu T, Shi C, Luo H, Wu Z, Xiong B, et al. Long non-coding RNA LUCAT1 promotes proliferation and invasion in gastric cancer by regulating miR-134-5p/YWHAZ axis. Biomed Pharmacother 2019;118:109201.

38. Han M, Dai D, Yousafzai NA, Wang F, Wang H, Zhou Q, et al. CXXC4 activates apoptosis through up-regulating GDF15 in gastric cancer. Oncotarget 2017;8:103557-67.

39. Chase A, Cross NC. Aberrations of EZH2 in cancer. Clin Cancer Res 2011;17:2613-8.

40. Benetatos L, Voulgaris E, Vartholomatos G, Hatzimichael E. Noncoding RNAs and EZH2 interactions in cancer: long and short tales from the transcriptome. Int J Cancer 2013;133:267-74. 